\title{
Características tempranas y predictores de la severidad del cuadro clínico en el trastorno del espectro autista
}

\author{
Early characteristics and predictors of clinical severity in \\ Autism Spectrum Disorder
}

\author{
Karol Patricia Gutiérrez-Ruiz $₫$ CvLAC-ORCID \\ Universidad Tecnológica de Bolívar \\ Colombia
}

Fecha correspondencia:

Recibido: enero 22 de 2018.

Aceptado: diciembre 20 de 2018.

Forma de citar:

Gutiérrez-Ruiz, K. (2019).

Características tempranas y

predictores de la severidad del

cuadro clínico en el Trastorno del

Espectro Autista. Rev.CES Psico,

12(2), $12-25$.

\section{Open access}

(c) Copyright

Licencia creative commons

Etica de publicaciones

Revisión por pares

Gestión por Open Journal System

DOI: http://dx.doi.org/10.21615/

cesp. 12.2 .2

ISSN: 2011-3080

Sobre los autores:

1. Doctora en Neuropsicología, Psicóloga. Docente investigadora de la Universidad Tecnológica de Bolívar. Directora del grupo de investigación Desarrollo, Salud y Desempeño Humano.

Comparte

\section{Resumen}

A nivel mundial se han realizado esfuerzos importantes para detectar el Trastorno del Espectro Autista (TEA) durante los primeros años de vida, dado los efectos positivos en el pronóstico del desarrollo de los niños diagnosticados. Objetivo. Describir los antecedentes del desarrollo y características tempranas de un grupo de niños colombianos con diagnóstico de TEA, y comparar estas características tempranas con las de un grupo de niños con desarrollo típico. Adicionalmente, relacionar variables del desarrollo con la severidad de los signos de TEA y analizar su valor predictivo. Método. Estudio no experimental de corte transversal en el que se aplicó el Q-CHAT a 40 padres de niños con diagnóstico temprano de autismo y 40 padres de niños con desarrollo típico, y se evaluó el desarrollo psicomotor de los menores, entre los 18 y 40 meses de edad, mediante el Inventario del Desarrollo Battelle Screening. Resultados. La primera preocupación de los padres del grupo de niños diagnosticados con TEA respecto al desarrollo de sus hijos fue alrededor de los 25 meses y la edad promedio de diagnóstico fue de 34,6 meses. Los signos tempranos de TEA más frecuentes y que mejor discriminan entre niños con desarrollo típico y con diagnóstico son aquellos que involucran atención conjunta. Adicionalmente, existe correlación negativa estadísticamente significativa entre la severidad de los signos de TEA y el desarrollo infantil en las áreas personal-social, cognitiva y comunicación expresiva; por lo cual, dificultades en el desarrollo de estas áreas serían predictores importantes de la severidad de los signos de TEA a edades tempranas.

Palabras claves: Trastorno del Espectro Autista, Autismo, Diagnóstico Precoz, Cribado, Desarrollo Infantil.

\section{Abstract}

Significant efforts have been made to detect Autistic Spectrum Disorder (ASD) during the first years of life, with positive effects on the prognosis of children. However, in countries such as Colombia early identification of ASD has not been easy. Objective. To describe the early characteristics and developmental antecedents in a group of Colombian children with diagnosis of ASD, comparing them with healthy children, and relating developmental 
variables to the severity of ASD signs by estimating their predictive value. Method. A cross-sectional study was carried out using a non-experimental design. The Q-CHAT was administered to 40 parents of children with early diagnosis of autism and 40 parents of children with typical development between 18 and 40 months. The psychomotor development was explored through the Battelle Screening Development Inventory. Results. The first concern of parents for their children development of was around 25 months old and the average age of diagnosis was 34.6 months. The most frequent early signs of ASD and that are better discriminated between children with typical development and those diagnosed with ASD are the ones that involve joint attention. Additionally, there is a strong negative correlation between the severity of ASD signs and child development in the personal-social, cognitive and expressive communication areas; these would be important predictors of the severity of the signs of ASD at early age.

Keywords: Autism Spectrum Disorder, Early Diagnosis, Screening, Child Development.

\section{Introducción}

El Trastorno del Espectro Autista (TEA) es un cuadro clínico de inicio en la infancia temprana que limita el desarrollo de habilidades sociales y comunicativas. Se caracteriza por la presencia de patrones restringidos, estereotipados y repetitivos de comportamientos, intereses y actividades (Gutiérrez-Ruiz, 2016), y sus síntomas pueden reconocerse desde el segundo año de vida (12-24 meses) e incluso antes si éstos son graves.

A nivel mundial se han realizado esfuerzos importantes para detectar el TEA durante los primeros años de vida, dado los efectos positivos en el pronóstico del desarrollo de los niños diagnosticados (Elder, Kreider, Brasher, \& Ansell, 2017). Estudios sobre características clínicas de niños con TEA en edades comprendidas entre el nacimiento y los dos años han evidenciado alteraciones en la conducta social y la comunicación caracterizadas por: poca exploración de objetos, tendencia a ignorar a las personas y a aislarse, interacción social reducida, contacto visual disminuido, déficit en la expresión de la sonrisa social, ausencia de respuesta al llamado por el nombre, alteración en protodeclarativos y en el uso de gestos, pobre modulación emocional e imitación, poca intención comunicativa, y alteración en el inicio espontáneo y respuesta a la atención conjunta (Baranek, Watson, Crais, \& Reznick, 2003; Clifford, Young, \& Williamson, 2007; Colgan et al., 2006; Maestro et al., 2005; Nadig et al., 2007; Young. Brewer, \& Pattison, 2003).

Con frecuencia el TEA es diagnosticado posterior a los tres años de edad (Fletcher-Watson et al., 2017). El rango de edad de diagnóstico oscila entre los 3 y los 10 años dependiendo de factores como la severidad de los síntomas e incluso el estrato socioeconómico (Daniels \& Mandell, 2014). Investigaciones en países europeos han mostrado una edad promedio de diagnóstico de 3,5 años (Salomone, Charman, McConachie, \& Warreyn, 2015) y el tiempo puede aumentar a cerca de nueve años en los casos de Síndrome de Asperger (Barbaro \& Dissanayake, 2009), actualmente considerado como TEA (APA, 2014).

Se estima que 1 de cada 68 niños ( 1 en 42 varones y 1 en 189 mujeres) en Estados Unidos es diagnosticado con TEA (Zablotsky, Black, Maenner, Schieve, \& Blumberg, 2015) y en Europa 1 por cada 100 (Autism Europe, 2015). En Colombia no existen cifras oficiales del número de personas con autismo (Talero et al., 2003). 
Pág 14

Aunque los indicadores clínicos del TEA sean universales, su expresión y severidad podría variar teniendo en cuenta factores socioculturales; e incluso, el autorreporte de los padres durante los procesos de seguimiento al desarrollo de los niños podría verse influenciado por estos factores, de manera que retrasos sutiles en el desarrollo pueden no ser detectados o pasar desapercibidos (Tek \& Landa, 2012).
Se han encontrado diferencias en la prevalencia y edad de diagnóstico, así como en la manifestación de conductas estereotipadas, el desarrollo cognitivo y del lenguaje entre niños con TEA de diferentes culturas (Chaidez, Hansen, \& Hertz-Picciotto, 2012; Tek \& Landa, 2012); y de síntomas del TEA relacionados con comunicación no verbal, resistencia al cambio e intereses restringidos (Matson et al., 2011). Así, aunque los indicadores clínicos del TEA sean universales, su expresión y severidad podría variar teniendo en cuenta factores socioculturales; e incluso, el autorreporte de los padres durante los procesos de seguimiento al desarrollo de los niños podría verse influenciado por estos factores, de manera que retrasos sutiles en el desarrollo pueden no ser detectados o pasar desapercibidos (Tek \& Landa, 2012).

Si bien, a nivel mundial la prevalencia del TEA ha aumentado y la edad de diagnóstico ha disminuido, en la población latina el TEA es infra diagnosticado debido a factores como la dificultad de acceso a servicios de salud y educativos, entre otros (Montiel-Nava, Chacín, \& González-Ávila, 2017). Algunas investigaciones han estudiado la población latina con TEA residente en Estados Unidos (Chaidez, et al., 2012; Daniels \& Mandell, 2014; Magaña, López, Aguinaga, \& Morton, 2013; Schieve et al., 2012), pero son pocos los estudios en los que se exploran las características y antecedentes del desarrollo de niños con diagnóstico temprano de TEA que viven en países latinoamericanos (Lara, Utria, \& Ávila-Toscano, 2012; Montiel-Nava et al., 2017; Villalba, 2013).

El objetivo de este trabajo es describir los antecedentes del desarrollo y características tempranas de un grupo de niños con diagnóstico de TEA, y comparar estas características tempranas con las de un grupo de niños con desarrollo típico. Esto con el fin de identificar cuáles de estas características son más frecuentes en los niños con TEA a temprana edad y establecer cuáles discriminan mejor entre los grupos, lo que es importante de cara a la identificación temprana del cuadro clínico en el ámbito socio-sanitario en nuestro medio. Adicionalmente, se pretende relacionar variables del desarrollo con la severidad de los signos de TEA y analizar su valor predictivo.

\section{Método}

\section{Participantes}

Se realizó un estudio no experimental de casos y controles, de corte transversal, con una muestra conformada por 40 niños (18-40 meses), 39 hombres y 1 mujer, con diagnóstico temprano de TEA y sus cuidadores principales; y 40 niños con desarrollo típico (18-40 meses), 21 hombres y 19 mujeres, y sus cuidadores principales. Los niños con diagnóstico de TEA conformaron el grupo clínico (casos) y los niños con desarrollo típico el grupo de comparación (control), apareados por edad y estrato socioeconómico.

Para conformar el grupo clínico, se realizó un muestreo no probabilístico por conveniencia identificando en la base de datos de los usuarios de las instituciones de salud Rehabilitar IPS y Pediacenter (Cartagena, Colombia), los niños con edades comprendidas entre 18 y 40 meses, con diagnóstico de TEA de acuerdo con los criterios del CIE-10 (Organización Mundial de la Salud -OMS-, 1992) o DSM-IV-TR (American Psychiatric Association -APA-, 2002). El diagnóstico fue realizado previamente por un equipo multidisciplinario conformado por un neuropediatra, un psicólogo clínico, un neuropsicólogo y un fonoaudiólogo. La presencia de cualquiera de las siguientes afecciones se consideró como criterio de exclusión: historia de hipoacusia severa, 
trastornos genéticos, trastornos metabólicos, síndrome de Guilles de la Tourette, encefalopatía, meningitis y discapacidad intelectual severa que cursara con síntomas similares a los encontrados en el TEA.

Los niños del grupo de control tenían historia de desarrollo neurológico típico y no presentaban antecedentes neurológicos y/o psiquiátricos. Para la selección de este grupo se realizó un muestreo no probabilístico por conveniencia de niños usuarios de un centro de estimulación infantil también de la ciudad de Cartagena, Colombia.

\section{Instrumentos}

Inventario del Desarrollo Battelle Screening (Newborg, Stock, \& Wneck, 1996). Prueba que evalúa las habilidades del desarrollo en niños entre el nacimiento y los ocho años. De aplicación individual, tipificada y compuesta por 96 ítems relacionados con las siguientes áreas del desarrollo: a) Personal/Social, que comprende la capacidad de realizar interacciones sociales significativas; b) Adaptativa, que evalúa las habilidades de autoayuda; c) Motora, que considera la capacidad de usar y controlar los músculos del cuerpo (fina y gruesa); d) Comunicación, que contiene la comunicación receptiva y expresiva; y e) Cognitiva, que aprecia habilidades y capacidades de tipo conceptual. Ofrece una puntuación por cada área y una puntuación total, y los resultados directos se transforman en edades equivalentes de desarrollo. Este inventario es una medida del desarrollo utilizada ampliamente en Colombia (Acosta, Moreno, \& Axpe, 2012; Campo-Ternera, 2010; Campo-Ternera, 2011; Flórez-Romero \& Arias-Velandia, 2010; Moraleda, Romero, \& Cayetano, 2013; Rubio, 2012).

Cuestionario Cuantitativo para la detección del Autismo en Niños pequeños (Q-CHAT por su abreviatura del inglés Quantitative Checklist for Autism in Toddlers) (Allison et al. 2008). Consta de 25 ítems dirigidos a los padres que permite cuantificar rasgos autistas de sus hijos en edades tempranas (desde los 18 meses con posibilidad de administrarlo hasta los 40 meses). El tiempo para completar el cuestionario está entre 5 y 10 minutos y se responde de acuerdo con una escala Likert de 5 puntos (0-4). Los datos preliminares sobre las propiedades psicométricas de la versión original del Q-CHAT (Allison et al., 2008) muestran una consistencia interna aceptable (alfa de Cronbach = $0.83)$ y una correlación test-retest de $0.82(n=330)$. El cuestionario ha sido traducido al español y adaptado en Colombia (Gutiérrez-Ruiz, 2016; Gutiérrez-Ruiz, Delgado, \& Prieto, 2017), y esta es la versión que se utilizó en el presente estudio.

\section{Procedimiento}

En primer lugar, se invitó a participar en la investigación a directivos de diferentes centros de atención pediátrica y de atención educativa a la primera infancia de la ciudad de Cartagena (Colombia); de los cuales se vincularon dos instituciones privadas: el Centro de Rehabilitación Integral de Cartagena - Rehabilitar IPS, especializado en atención neuropediátrica, y Pediacenter, en atención pediátrica.

Una vez informado sobre los objetivos y condiciones del estudio, los padres de los niños diligenciaron un cuestionario sociodemográfico y de antecedentes del desarrollo, y el Q-CHAT; en tanto que los menores fueron evaluados mediante la aplicación del Inventario del Desarrollo Battelle Screening.

\section{Consideraciones éticas}

El presente estudio contó con el aval del Comité de investigaciones de la Universidad Tecnológica de Bolívar. Todos los sujetos participaron voluntariamente y firmaron un consentimiento informado, y se les informó que podían desistir en cualquier momento 
Pág 16

La primera preocupación de los padres por el desarrollo de sus hijos surgió en promedio a los 25 meses $(\mathrm{DE}=7.7)$ y se relacionó con retraso en el desarrollo del habla. Los niños de este grupo clínico recibieron el diagnóstico en promedio a los 34.6 meses (DE = 8.2), siendo más frecuente el de Trastorno generalizado del desarrollo no especificado (62.5\%), seguido de Trastorno autista (35\%) y Síndrome de Asperger (2.5\%). de su participación. De igual manera, se comunicó que los datos serían codificados mediante números para evitar la identificación de los participantes y de su carácter confidencial.

\section{Análisis de datos}

Los datos correspondientes a las variables cualitativas como factores sociodemográficos y antecedentes del desarrollo se analizaron por medio de medidas de frecuencias absolutas y relativas expresadas en porcentajes; y los correspondientes a las variables cuantitativas por medio de medidas de tendencia central como media y mediana, y de dispersión como el rango y la desviación estándar. Estos análisis permitieron identificar los antecedentes del desarrollo y las principales características clínicas del grupo de niños con TEA.

Para las comparaciones de los datos entre el grupo clínico y el de control respecto a la valoración de características tempranas de TEA mediante el Q-CHAT se utilizó el estadístico de prueba U de Mann-Whitney, esto con el fin de identificar cuáles de estas características discriminan mejor entre los grupos. Adicionalmente, se correlacionaron variables del desarrollo (área personal social, adaptativa, motora, comunicativa y cognitiva) con la severidad de los signos de TEA y se analizó su valor predictivo mediante un modelo de regresión lineal. La severidad de los signos de TEA se estimó mediante la puntuación global del Q-CHAT. Los análisis se realizaron con el paquete estadístico para las ciencias sociales (SPSS) versión 24.0 para Windows.

\section{Resultados}

Mediante el cuestionario sociodemográfico y de antecedentes del desarrollo diligenciado por los padres de los menores se obtuvo la siguiente información sobre las características y edad de adquisición de logros del desarrollo de los niños:

En los niños con diagnóstico de TEA estudiados se observó predominio del género masculino $\left(\mathrm{N}_{\text {mujeres }}=1, \mathrm{~N}_{\text {varones }}=39\right)$. La primera preocupación de los padres por el desarrollo de sus hijos surgió en promedio a los 25 meses $(D E=7.7)$ y se relacionó con retraso en el desarrollo del habla. Los niños de este grupo clínico recibieron el diagnóstico en promedio a los 34.6 meses $(D E=8.2)$, siendo más frecuente el de Trastorno generalizado del desarrollo no especificado (62.5\%), seguido de Trastorno autista (35\%) y Síndrome de Asperger (2.5\%). El 68.7\% de los niños fue producto del primer embarazo, $77.1 \%$ de los embarazos fue de 40 semanas de gestación (en ningún caso se reportó prematuridad) y $82.9 \%$ de los partos fueron por cesárea. Entre los antecedentes obstétricos más frecuentes se destacan abortos previos y amenaza de aborto (11.4\%) y aumento de la presión arterial de la madre (11.4\%). En cuanto a los antecedentes personales de los niños, en dos casos se reportó trauma craneoencefálico leve (TCE) antes de los 18 meses, en los restantes no se encontró antecedentes de importancia (ver Tabla 1). El desarrollo motor durante el primer año fue en términos generales normal. En lo que se refiere al desarrollo del lenguaje expresivo (balbuceo, palabras y frases), la edad de adquisición se presentó en promedio por encima de lo esperado, es decir, evidenció retraso; y algunos niños no habían desarrollado lenguaje expresivo en el momento de la valoración. 
Tabla 1. Antecedentes clínicos relevantes del grupo clínico (niños con diagnóstico de TEA)

\begin{tabular}{lc}
\hline Antecedentes familiares & $\%$ \\
\hline Retraso en el lenguaje & 2.9 \\
\hline Antecedentes obstétricos & $\%$ \\
\hline Embarazo de alto riesgo & 5.7 \\
Miomectomía previa & 2.9 \\
Hipertensión arterial & 8.6 \\
Amenaza de aborto & 5.7 \\
Placenta previa & 2.9 \\
Pre-eclampsia & 2.9 \\
Infección vaginal frecuente & 2.9 \\
\hline Gestación & \\
\hline Tiempo promedio & 39.51 \\
Pretérmino 37 semanas & 0 \\
\hline Tipo de parto & \\
\hline Vaginal & 17.14 \\
Cesárea & 82.86 \\
\hline Antecedentes personales & 2.9 \\
\hline Cataratas congénitas & 2.9 \\
Hipoxia & 5.7 \\
Bajo peso & 5.7 \\
\hline Epilepsia & 2.9 \\
\hline & 2.9 \\
\hline Cirugía de adenoides & 2.9 \\
\hline
\end{tabular}

Tabla 2. Edad de adquisición de logros del desarrollo en el grupo clínico (niños con diagnóstico de TEA)

\begin{tabular}{lcccccc}
\hline $\begin{array}{c}\text { Aspecto del } \\
\text { desarrollo }\end{array}$ & $\begin{array}{c}\text { Media } \\
\text { (meses) }\end{array}$ & $\begin{array}{c}\text { Mínimo } \\
\text { (meses) }\end{array}$ & $\begin{array}{c}\text { Máximo } \\
\text { (meses) }\end{array}$ & $\begin{array}{c}\text { Adquisición tardía } \\
\text { (meses) \% }\end{array}$ & $\begin{array}{c}\text { No adquirido } \\
\text { (\%) }\end{array}$ & $\begin{array}{c}\text { Retroceso en el } \\
\text { lenguaje (\%) }\end{array}$ \\
\hline Gateo & 7.9 & 6 & 10 & $>10(0)$ & 14.28 & \\
Marcha & 12.29 & 6 & 20 & $>18(2.9)$ & & 25.71 \\
Balbuceo & 11.6 & 4 & 40 & & 11.4 & \\
Palabras & 21.71 & 8 & 40 & $>18(45)$ & & \\
\hline
\end{tabular}

Fuente: Cuestionario sociodemográfico y de antecedentes del desarrollo diligenciado por los padres.

En el grupo de niños con desarrollo típico se presentó predominio del género masculino $\left(N_{\text {mujeres }}=19, N_{\text {varones }}=21\right)$, edad promedio de $28(D E=6.4)$ meses, $75 \%$ de los embarazos fue de 40 semanas de gestación y $70 \%$ de los partos fueron por cesárea. El 95\% no refiere antecedentes obstétricos de importancia, en un caso se informó pre-eclampsia y, en otro, disminución en la motilidad del feto. En cuanto a antecedentes perinatales, en un caso se informó cianosis. 
Al comparar los resultados obtenidos por los grupos en la valoración de características tempranas de TEA mediante el Q-CHAT (ver Tabla 3), se observó que existen diferencias estadísticamente significativas de modo que en los niños del grupo clínico se presentó mayor dificultad para establecer contacto visual $(U=302.5, p=.000, r=0.587)$ y mirar cuando le llaman por su nombre $(U=118.0, p=.000, r=0.807)$, raramente pueden otras personas entender al niño cuando habla ( $U=95.0, p=.000, r=0.79)$, pocas veces señala para compartir intereses $(U=386.5, p=.000, r=0.48)$ y cuando se enfrenta a una situación desconocida sólo en ocasiones mira al adulto espontáneamente para comprobar su reacción (atención conjunta) $(U=280.5, p=.001, r=0.59)$. Pueden decir menos de 10 palabras $(U=236.0, p=.000, r=0.64)$, el juego simbólico es escaso o nulo $(U=290.0$, $p=.000, r=0.57)$ muestran menor conducta empática cuando alguien está visiblemente angustiado $(U=319.0, p=.000, r=0.55)$ y usan ocasionalmente gestos simples $(U=323.5$, $p=.000, r=0.56$ ). Algunos de los signos de TEA evaluados se expresan sutilmente en la población con diagnóstico, sin embargo, existen diferencias estadísticamente significativas entre sujetos con TEA y con desarrollo típico en estos signos: los niños con TEA miran algunas veces por día hacia donde el padre está mirando $(U=476.5, p=.001$, $r=0.37$ ) y puede que muestren leve dificultad para adaptarse cuando la rutina cambia o cuando las cosas están fuera de lugar $(U=579.5, p=.030, r=0.24)$. Es interesante notar que conductas repetitivas e intereses restringidos como caminar en punta de pies, ecolalias, movimientos raros con los dedos de las manos cerca de los ojos y hacer girar repetitivamente objetos no discriminan adecuadamente entre niños con TEA y niños con un desarrollo típico a temprana edad.

Tabla 3. Comparación de características tempranas de TEA mediante el Q-CHAT

\begin{tabular}{|c|c|c|}
\hline Ítems del Q-CHAT & $\begin{array}{l}\text { U de Mann- } \\
\text { Whitney }\end{array}$ & $\boldsymbol{p}$ \\
\hline 11. ¿Su hijo/a le mira cuando usted lo llama por su nombre? & 118.0 & .000 \\
\hline 12. ¿Cuán fácil le resulta establecer contacto visual con su hijo? & 302.5 & .000 \\
\hline 13. Cuando su hijo/a está jugando solo/a, ¿alinea objetos? & 688.5 & .272 \\
\hline 14. ¿Pueden otras personas entender fácilmente a su hijo/a cuando habla? & 95.0 & .000 \\
\hline 15. ¿Señala su hijo/a para indicar que quiere algo? & 623.5 & .058 \\
\hline 16. ¿Señala su hijo/a para compartir un interés con usted? & 386.5 & .000 \\
\hline $\begin{array}{l}\text { 17. ¿Cuánto tiempo puede un objeto giratorio mantener el interés de su } \\
\text { hijo/a? }\end{array}$ & 524.5 & .005 \\
\hline 18. ¿Cuántas palabras puede decir su hijo/a? & 236.0 & .000 \\
\hline 19. ¿Su hijo/a juega a "hacer como si"? & 290.0 & .000 \\
\hline 110. ¿Su hijo/a mira hacia donde usted está mirando? & 476.5 & .001 \\
\hline 111. ¿Con qué frecuencia su hijo/a huele o lame objetos inusuales? & 686.5 & .256 \\
\hline $\begin{array}{l}\text { 112. ¿Agarra su hijo/a su mano y la pone sobre un objeto cuando quiere } \\
\text { que usted lo use? }\end{array}$ & 647.0 & .108 \\
\hline 113. ¿Camina su hijo/a en puntas de pie? & 439.5 & .000 \\
\hline $\begin{array}{l}\text { 114. ¿Cuán fácil le resulta a su hijo/a adaptarse cuando su rutina cambia o } \\
\text { cuando las cosas están fuera de lugar? }\end{array}$ & 579.5 & .030 \\
\hline $\begin{array}{l}\text { 115. ¿Si usted o alguien más en la familia está visiblemente angustiado/a, } \\
\text { muestra su hijo/a signos de querer consolarlo/a? }\end{array}$ & 319.0 & .000 \\
\hline 116. ¿Hace su hijo/a la misma cosa una y otra vez? & 789.0 & .911 \\
\hline
\end{tabular}




\begin{tabular}{lcc}
\hline \multicolumn{1}{c}{ Ítems del Q-CHAT } & $\begin{array}{c}\text { U de Mann- } \\
\text { Whitney }\end{array}$ & p \\
\hline I17. Describiría las primeras palabras de su hijo/a como & 611.5 & .047 \\
\hline I18. ¿Repite su hijo cosas que escucha? & 442.0 & .000 \\
\hline I19. ¿Utiliza su hijo/a gestos simples? & 323.5 & .000 \\
\hline I20. ¿Realiza su hijo/a movimientos raros con los dedos de la mano cerca & 574.5 & .020 \\
de sus ojos? & 280.5 & .001 \\
\hline I21. Cuando su hijo/a se enfrenta a una situación desconocida, ¿le mira a & 795.0 & .961 \\
\hline la cara espontáneamente para comprobar sus reacción? & 566.5 & .017 \\
\hline $\begin{array}{l}\text { I22. Durante cuánto tiempo pueden solo uno o dos objetos mantener el } \\
\text { interés de su hijo/a? }\end{array}$ & 644.5 & .124 \\
\hline I23. ¿Se hijo/a hacer girar objetos repetitivamente? & 713.0 & .338 \\
\hline I24. ¿Parece su hijo/a ser demasiado sensible al ruido? & & \\
\hline I25. ¿Su hijo es de mirar fijo a la nada sin un propósito aparente? & & \\
\hline
\end{tabular}

Al comparar los logros del desarrollo de los niños de ambos grupos mediante el Inventario del Desarrollo Battelle Screening, se encontró que los niños con TEA presentan un desarrollo significativamente por debajo del esperado para su edad en todas las áreas en comparación con niños con desarrollo típico: personal-social ( $U=73.0, p=.000$, $r=0.79)$, adaptativa $(U=376.5, p=.000, r=0.45)$, comunicación $(U=121.0, p=.000, r=0.73)$, motora $(U=294.0, p=.000, r=0.55)$ y cognitiva $(U=347.0, p=.000, r=0.49)$. Las áreas en las que se encuentra mayor discrepancia entre grupos fueron el área personal-social y comunicativa.

El modelo de regresión lineal mostró relación entre la variable severidad de los signos del TEA, estimada mediante la puntuación total en el Q-CHAT y la puntuación en las áreas personal social $(r=-.601 . p=.000)$, cognitiva $(r=-.206, p=.033)$, comunicación expresiva $(r=-.531, p=.000)$ y motora $(r=-0.228, p=.021)$ del Inventario del Desarrollo Battelle Screening. La puntuación obtenida en estas áreas sería, por tanto, un predictor importante de la severidad de los signos del TEA, con un coeficiente de determinación de .525 y error cuadrático medio de 52.43, de modo que a menor nivel de desarrollo en estas áreas mayor es la expresión de signos asociados al TEA en edades tempranas. En la Tabla 4 se muestran los coeficientes tipificados y sus valores de probabilidad.

Tabla 4. Predictores importantes de la severidad de los signos de TEA. Coeficientes tipificados y probabilidad

\begin{tabular}{lccccc}
\hline & $\boldsymbol{B}$ & SE B & $\boldsymbol{B}$ estandarizado & $\boldsymbol{t}$ & $\boldsymbol{p}$ \\
\hline Constante & 37.05 & 6.946 & & 5.335 & .000 \\
Personal social & -1.160 & .253 & -.612 & -4.693 & .000 \\
Cognitiva & 1.259 & .368 & .448 & 3.421 & .001 \\
Expresiva & -2.632 & .720 & -.588 & -3.653 & .000 \\
Motora & 1.042 & .498 & .277 & 2.090 & .040 \\
\hline
\end{tabular}


Las características tempranas del TEA más frecuentes y que mejor discriminan entre niños con desarrollo típico y niños con diagnóstico son aquellas que involucran interacción y comunicación social.

\section{Discusión}

La realización de estudios en niños con diagnóstico temprano del TEA en Colombia es un primer paso para identificar posibles variaciones en el reporte de sus síntomas que pudieran estar asociados a nuestro contexto (Tek \& Landa, 2012); lo que permite mayor efectividad en los procesos de detección temprana de TEA.

Conforme al objetivo de describir los antecedentes del desarrollo y características tempranas de un grupo de niños con diagnóstico de TEA, en este estudio se identifica que la primera preocupación de los padres respecto al desarrollo de sus hijos surge en promedio a los 25 meses y se relaciona principalmente con el retraso en el desarrollo del lenguaje. Respecto a la edad de reconocimiento de signos del TEA, las investigaciones apuntan a que la mayoría de los padres de niños con TEA notan anormalidades durante el curso del desarrollo en los primeros dos años de vida (Baghdadli, Picot, Pascal, Pry, \& Aussilloux, 2003; Chawarska et al., 2007; Zablotsky et al., 2017). En países latinoamericanos como en Venezuela se ha reportado que las primeras preocupaciones surgen en promedio a los 17 meses (Montiel-Nava et al., 2017) y en Brasil a los 23 meses (Ribeiro, Paula, Bordini, Mari, \& Caetano, 2017).

El tiempo transcurrido desde las primeras preocupaciones de los padres y el diagnóstico médico del TEA dado a los niños es variable; así, en países de bajos y medianos ingresos, la edad de los niños cuando se les da el diagnóstico suele estar en el rango de entre 45 y 57 meses, por encima de países de altos ingresos (36 y 42 meses). En países latinoamericanos como Venezuela el diagnóstico se realiza en promedio a los 54 meses de edad de los niños (Montiel-Nava et al., 2017), en Brasil a los 59 meses (Ribeiro et al., 2017) y en Colombia a los 45 meses (Talero-Gutiérrez. Rodríguez, De la Rosa, Morales, \& Vélez-Van-Meerbeke, 2012). En el presente estudio el diagnóstico a los niños del grupo clínico se realizó en promedio a los 34 meses, edad que se encuentra por debajo de la media mundial de 42 meses (Salomone et al., 2015); esta situación probablemente obedece a una mayor conciencia sobre el TEA tanto de los cuidadores como de los profesionales de la salud en los últimos años (Alcantud, Alonso, \& Mata, 2016), lo que ha facilitado la identificación de signos de alerta temprana. Lo anterior, sugiere que es posible disminuir el tiempo de realización del diagnóstico de TEA y una de las estrategias que lo facilita es atender a las sospechas tempranas de los padres sobre anormalidades en el desarrollo de sus hijos (Zablotsky et al., 2017).

De acuerdo con los resultados del presente estudio, las características tempranas del TEA más frecuentes y que mejor discriminan entre niños con desarrollo típico y niños con diagnóstico son aquellas que involucran interacción y comunicación social. A continuación se mencionan cada una de estas características y se citan estudios previos con hallazgos similares: atención conjunta o compartida alterada (pocas veces señala para compartir intereses y cuando se enfrenta a una situación desconocida sólo en ocasiones mira al adulto espontáneamente para comprobar su reacción) (Baranek et al., 2003; Wetherby, Watt, Morgan, \& Shumway, 2007), dificultad para establecer contacto visual y poca respuesta al llamado por el nombre (Baranek et al., 2003; Wetherby et al., 2007; Zwaigenbaum et al., 2005), raramente otras personas pueden entender al niño cuando habla (Mitchell et al., 2006; Zwaigenbaum et al., 2005), poca empatía (Saint-Georges et al., 2010), retraso en el desarrollo del lenguaje expresivo con un número reducido de palabras (Mitchell et al., 2006; Zwaigenbaum et al., 2005), el juego simbólico es escaso o nulo (Saint-Georges et al., 2010) y uso ocasional de gestos simples (Baranek et al., 2003, Watson et al., 2007). 
Se encontró una correlación negativa estadísticamente significativa entre la severidad de los signos clínicos del TEA y el desarrollo infantil en las áreas personal-social, cognitiva, comunicación expresiva y motora. Entre éstas, la variable más importante para predecir la severidad del TEA es el desarrollo personal-social, seguido del desarrollo cognitivo y el lenguaje expresivo.
Estos hallazgos son coherentes con los de otros estudios que catalogan las conductas sociales (atención social y comunicación social) como el mejor indicador de diagnóstico diferencial (Baranek et al., 2003, Watson et al., 2007). En el presente estudio las conductas de imitación y síntomas motores y sensoriales de TEA como alteraciones en el procesamiento sensorial (intereses sensoriales inusuales y reacciones atípicas ante estímulos sensoriales), los patrones restrictivos de comportamiento y las conductas repetitivas, no discriminan adecuadamente entre niños con diagnóstico temprano de TEA y niños con desarrollo típico (Baranek et al., 2003; Swinkels et al., 2006). En consecuencia, puede señalarse que estos síntomas, que con frecuencia se consideran en los instrumentos de cribado de TEA desarrollados en Estados Unidos y Europa (Allison et al., 2008; Robins, Fein, Barton, \& Green, 2001; Stone, Coonrod, \& Ousley, 2000; Swinkels et al., 2006), no permiten identificar adecuadamente niños con TEA y con desarrollo típico en nuestro contexto (Colombia), lo que es importante considerar en la práctica clínica.

Se encontró una correlación negativa estadísticamente significativa entre la severidad de los signos clínicos del TEA y el desarrollo infantil en las áreas personal-social, cognitiva, comunicación expresiva y motora. Entre éstas, la variable más importante para predecir la severidad del TEA es el desarrollo personal-social, seguido del desarrollo cognitivo y el lenguaje expresivo. Estas observaciones son coherentes con el hecho de que el aspecto más afectado en el autismo es la reciprocidad social, lo que incide en el funcionamiento de los niños en actividades de la vida diaria propias de su edad y en procesos de escolarización (Allison et al., 2008; Allison, Auyeung, \& Baron-Cohen, 2012; Clifford et al., 2007; Clifford \& Dissanayake, 2008; Nadig et al., 2007). De igual manera, estos hallazgos coinciden con los resultados del estudio de Sanz, Guijarro y Sánchez (2007), quienes encontraron que sujetos de población española en edades comprendidas entre 30 y 32 meses con diagnóstico de TEA alcanzaban cocientes de desarrollo muy bajos en las áreas comunicativa (receptiva y expresiva) y personal-social.

Finalmente, se pone en evidencia que a medida que aumenta la severidad del TEA disminuyen las puntuaciones en la evaluación del desarrollo psicomotor de los niños. Se podría pensar en el Inventario del Desarrollo Battelle Screening como un instrumento de apoyo en el cribado de TEA, esperando encontrar menores cocientes del desarrollo en las áreas personal-social, cognitiva y comunicación expresiva, a medida que hay mayor presencia de signos de TEA. Incluir la exploración de estas áreas en las consultas rutinarias de los niños es fundamental para facilitar la detección temprana del TEA y mitigar su impacto en la vida del paciente, su familia y en el sistema de salud. El retraso en estas áreas debe alertar a los profesionales de la salud sobre la necesidad de utilizar herramientas de cribado de TEA para corroborar la sospecha de alteración en el desarrollo comunicativo y social en la infancia, y derivar a centros de diagnóstico especializados en caso de ser necesario. Así mismo, este estudio ha permitido explorar la utilidad del Battelle Screening como instrumento de evaluación del desarrollo que alerta sobre signos de TEA a temprana edad y permite hacer seguimiento en ámbitos no solo clínico asistenciales sino también comunitarios, lo que facilita la remisión oportuna.

Cabe mencionar que, si bien los datos son informativos, el tamaño muestral del presente estudio no permite hacer inferencias a la población general, por lo que se sugiere que futuras investigaciones amplíen el tamaño de la muestra e incluyan niños de diferentes grupos poblacionales y estratos socioeconómicos. Se sugiere, además, controlar la variable sexo incluyendo igual número de participantes varones 
y mujeres en los grupos clínico y de comparación. Por último, se recomienda que futuras investigaciones tengan en cuenta los criterios del DSM-5 con el fin de confirmar la estabilidad de los resultados pese a las variaciones recientes en los criterios diagnósticos.

\section{Referencias}

Acosta, V., Moreno, A., \& Axpe, A. (2012). Implicaciones clínicas del diagnóstico diferencial temprano entre Retraso de Lenguaje $(R L)$ y Trastorno Específico del Lenguaje (TEL). Universitas Psychologica, 11, 279-291. Disponible en http://www.scielo.org. co/scielo.php?script=sci arttext\&pid=S1657-92672012000100023

Alcantud, M. F., Alonso, E. Y., \& Mata, I.S. (2016). Prevalencia de los trastornos del espectro autista: revisión de datos. Siglo Cero. Revista Española sobre Discapacidad Intelectual, 47(4), 7-26. doi: http://dx.doi.org/10.14201/scero2016474726

Allison, C., Auyeung, B., \& Baron-Cohen, S. (2012). Toward brief "Red Flags" for autism screening: the short autism spectrum quotient and the short quantitative checklist in 1,000 cases and 3,000 controls. Journal of the American Academy of Child and Adolescent Psychiatry. 51, 202-12. doi: https://doi.org/10.1016/j. jaac.2011.11.003

Allison, C., Baron-Cohen, S., Wheelwright, S., Charman, T., Richler, J., Pasco, G. ... Brayne. (2008). The Q-CHAT (Quantitative Checklist for Autism in Toddlers): A normally distributed quantitative measure of autistic traits at 18-24 months of age. Preliminary report. Journal of Autism and Developmental Disorders, 38, 1414-25. doi: https://doi.org/10.1007/s10803-007-0509-7

American Psychiatric Association (APA). (2002). Manual Diagnóstico y Estadístico de los Trastornos Mentales $4^{a}$. versión revisada. Barcelona: MASSON S.A.

Asociación Americana de Psiquiatría (APA). (2014). Manual diagnóstico y estadístico de los trastornos mentales: DSM-5. Arlington: Editorial medica panamericana.

Autism Europe. (2015). Prevalence rate of Autism. Belgium: Autism-Europe.

Baghdadli, A., Picot, M.C., Pascal, C., Pry, R., \& Aussilloux, C. (2003). Relationship between age of recognition of first disturbances and severity in young children with autism. European Child \& Adolescent Psychiatry, 12(3),122-127. doi: https://doi.org/10.1007/s00787-003-0314-6

Baranek, G.T., Watson, L.R., Crais, E.R., \& Reznick, J.S. (2003). First Year Inventory (FYI) (Version 2.0). Chapel Hill, NC: The University of North Carolina at Chapel Hill.

Barbaro, J., \& Dissanayake, C. (2009). Autism spectrum disorders in infancy and toddlerhood: a review of the evidence on early signs, early identification tools, and early diagnosis. Journal of Developmental \& Behavioral Pediatrics, 30(5), 447-59. doi: https://doi.org/10.1097/DBP.0b013e3181ba0f9f

Campo-Ternera, L. (2010). Importancia del desarrollo motor en relación con los procesos evolutivos del lenguaje y la cognición en niños de 3 a 7 años de la ciudad de Barranquilla (Colombia). Revista Salud Uninorte, 26, 65-76. Disponible en http:// rcientificas.uninorte.edu.co/index.php/salud/article/viewArticle/106/5793

Campo-Ternera, L. (2011). Características del desarrollo adaptativo en niños de 3 a 7 años de la ciudad de Barranquilla. Psychologia. Avances de la Disciplina, 5, 95104. Disponible en https://www.redalyc.org/pdf/2972/297224105008.pdf

Chaidez, V., Hansen, R.L., \& Hertz-Picciotto, I. (2012). Autism spectrum disorders in Hispanics and non-Hispanics. Autism 16(4), 381-397. doi: https://doi. org/10.1177/1362361311434787

Chawarska, K., Paul, R., Klin, A., Hannigen, S., Dichtel, L.E., \& Volkmar, F. (2007). Parental recognition of developmental problems in toddlers with autism spectrum disorders. Journal of Autism and Developmental Disorders. 37(1), 62-72. doi: https://doi. org/10.1007/s10803-006-0330-8 
Clifford, S., Young, R., \& Williamson, P. (2007). Assessing the early characteristics of autistic disorder using video analysis. Journal of Autism and Developmental Disorders, 37(2), 301-13. doi: https://doi.org/10.1007/s10803-006-0160-8

Clifford, S.M., \& Dissanayake, C. (2008). The early development of joint attention in infants with autistic disorder using home video observations and parental interview. Journal of Autism and Developmental Disorders, 38, 791-805. doi: https:// doi.org/10.1007/s10803-007-0444-7

Colgan, S., Lanter, E., McComish, C., Watson, L., Crais, E., \& Baranek, G. (2006). Analysis of Social Interaction Gestures in Infants with Autism. Child Neuropsychology, 12(4- 5), 307-19. doi: https://doi.org/10.1080/09297040600701360

Daniels, A. M., \& Mandell, D.S. (2014). Explaining differences in age at autism spectrum disorder diagnosis: a critical review. Autism, 18(5), 583-97. doi: https://doi. org/10.1177/1362361313480277

Elder, J. H., Kreider, C. M., Brasher, S. N., \& Ansell, M. (2017). Clinical impact of early diagnosis of autism on the prognosis and parent-child relationships. Psychology Research and Behavior Management, 10, 283. doi: https://doi.org/10.2147/PRBM. $\underline{\mathrm{S} 117499}$

Fletcher-Watson, S., Apicella, F., Auyeung, B., Beranova, S., Bonnet-Brilhault, F., Canal-Bedia, R.,... Farroni, T. (2017). Attitudes of the autism community to early autism research. Autism, 21(1), 61-74. doi: https://doi.org/10.1177/1362361315626577

Flórez-Romero, R., \& Arias-Velandia, N. (2010). Evaluación de conocimientos previos del aprendizaje inicial de lectura. Magis, Revista Internacional de Investigación en Educación, 2, 329-343. Disponible en: https://revistas.javeriana.edu.co/index. php/MAGIS/article/view/3516

Gutiérrez-Ruiz, K., Delgado, A.R., \& Prieto, G. (2017). Rasch Analysis of the Q-CHAT in Colombian Toddlers with Autism Spectrum Disorder. Current Psychology, 1-5. doi: https://doi.org/10.1007/s12144-017-9596-6

Gutiérrez-Ruiz, K. (2016). Identificación temprana de trastornos del espectro autista. Acta Neurológica Colombiana, 32(3), 238-247. Disponible en http://www.scielo.org.co/pdf/anco/v32n3/v32n3a11.pdf

Lara, D., Utria, O., \& Ávila-Toscano, J. (2012). Pre-, peri-and postnatal risk factors associated with gender in children with autism. International Journal of Psychological Research, 5(2), 77-90. Disponible en http://www.scielo.org.co/pdf/ i.jpr/v5n2/v5n2a09.pdf

Maestro, S., Muratori, F., Cesari, A., Cavallaro, M.C., Paziente, A., Pecini, C., ... Sommario, C. (2005). Course of Autism Signs in the First Year of Life. Psychopathology, 38(1), 26-31. doi: https://doi.org/10.1159/000083967

Magaña, S., Lopez, K., Aguinaga, A., \& Morton, H. (2013). Access to diagnosis and treatment services among Latino children with autism spectrum disorders. Intellectual and Developmental Disabilities, 51(3), 141-153. doi: https://doi. org/10.1352/1934-9556-51.3.141

Matson, J. L., Worley, J. A., Fodstad, J. C., Chung, K. M., Suh, D., Jhin, H. K., ... Furniss, F. (2011). A multinational study examining the cross cultural differences in reported symptoms of autism spectrum disorders: Israel, South Korea, the United Kingdom, and the United States of America. Research in Autism Spectrum Disorders, 5(4), 1598-1604. Disponible en: https://yonsei.pure.elsevier.com/en/publications/a-multinational-study-examining-the-cross-cultural-differences-in

Mitchell, S., Brian, J., Zwaigenbaum, L., Roberts, W., Szatmari, P., Smith, I., \& Bryson, S. (2006). Early language and communication development of infants later diagnosed with autism spectrum disorder. Journal of Developmental \& Behavioral Pediatrics, 27(2), S69-S78. Disponible en: https://www.ncbi.nlm.nih.gov/pubmed/16685188 
Montiel-Nava, C., Chacín, J. A., \& González-Ávila, Z. (2017). Age of diagnosis of autism spectrum disorder in Latino children: The case of Venezuelan children. Autism, 21(5), 573-580. doi: https://doi.org/10.1177/1362361317701267

Moraleda, E., Romero, M., \& Cayetano, M. (2013). La parálisis cerebral como una condición dinámica del cerebro: un estudio secuencial del desarrollo de niños hasta los 6 años de edad. Universitas Psychologica, 12, 119-127. Disponible en http://www.scielo.org.co/pdf/rups/v12n1/v12n1a12.pdf

Nadig, A. S., Ozonoff, S., Young, G. S., Rozga, A., Sigman, M., \& Rogers, S. J. (2007). A prospective study of response to name in infants at risk for autism. Archives of Pediatrics \& Adolescent Medicine, 161(4), 378-383. doi: https://doi.org/10.1001/ archpedi.161.4.378

Newborg, J., Stock, J., \& Wneck, L. (1996). Inventario del Desarrollo Battelle. Madrid: TEA. Organizacion Mundial de la Salud (OMS). (1992). Cie 10. Trastornos mentales y del comportamiento: descripciones clínicas y pautas para el diagnóstico. Madrid: Meditor.

Ribeiro, S. H., Paula, C. S. D., Bordini, D., Mari, J. J., \& Caetano, S. C. (2017). Barriers to early identification of autism in Brazil. Revista Brasileira de Psiquiatría, (AHEAD), 0-0. doi: https://doi.org/10.1590/1516-4446-2016-2141

Robins, D. L., Fein, D., Barton, M. L., \& Green, J. A. (2001). The Modified Checklist for Autism in Toddlers: an initial study investigating the early detection of autism and pervasive developmental disorders. Journal of Autism and Developmental Disorders, 31(2), 131-144. Disponible en https://www.ncbi.nlm.nih.gov/pubmed/11450812

Rubio, M. (2012). Inventario de desarrollo como instrumento de ayuda diagnóstica en niños con dificultades en la participación de actividades escolares. Revista Colombiana de Psiquiatría, 41, 139-149. doi: https://doi.org/10.1016/S00347450(14)60073-1

Saint-Georges, C., Cassel, R. S., Cohen, D., Chetouani, M., Laznik, M. C., Maestro, S., \& Muratori, F. (2010). What studies of family home movies can teach us about autistic infants: A literature review. Research in Autism Spectrum Disorders, 4(3), 355-366. doi: https://doi.org/10.1016/j.rasd.2009.10.017

Salomone, E., Charman, T., McConachie, H., \& Warreyn, P. (2015). Child's verbal ability and gender are associated with age at diagnosis in a sample of young children with ASD in Europe. Child: Care, Health and Development, 42(1), 141-145. doi: https://doi. org/10.1111/cch.12261

Sanz, Y., Guijarro, T., \& Sánchez, V. (2007). Inventario del Desarrollo Battelle como instrumento de ayuda diagnóstica en el autismo. Revista de la Asociación Española de Neuropsiquiatría, 27, 303-317. Disponible en https://www.redalyc.org/ pdf/2650/265019653004.pdf

Schieve, L. A., Rice, C., Yeargin-Allsopp, M., Boyle, C. A., Kogan, M. D., Drews, C., \& Devine, 0. (2012). Parent-reported prevalence of autism spectrum disorders in US-born children: an assessment of changes within birth cohorts from the 2003 to the 2007 National Survey of Children's Health. Maternal and Child Health Journal, 16(1), 151-157. doi: https://doi.org/10.1007/s10995-012-1004-0

Stone, W. L., Coonrod, E. E., \& Ousley, O. Y. (2000). Brief report: screening tool for autism in two-year-olds (STAT): development and preliminary data. Journal of autism and developmental disorders, 30(6), 607-612. doi: https://doi. org/10.1023/A:1005647629002

Swinkels, S. H., Dietz, C., van Daalen, E., Kerkhof, I. H., van Engeland, H., \& Buitelaar, J. K. (2006). Screening for autistic spectrum in children aged 14 to 15 months. I: the development of the Early Screening of Autistic Traits Questionnaire (ESAT). Journal of Autism and Developmental Disorders, 36(6), 723-732. doi: https://doi. org/10.1007/s10803-006-0115-0 
Talero, C., Martínez, L. E., Mercado, M., Ovalle, J. P., Velásquez, A., \& Zarruk, J. G. (2003). Autismo: estado del arte. Revista Ciencias de la Salud, 1(1), 68-85. Disponible en https://revistas.urosario.edu.co/index.php/revsalud/article/view/793

Talero-Gutiérrez, C., Rodríguez, M., De La Rosa, D., Morales, G., \& Vélez-Van-Meerbeke, A. (2012). Profile of children and adolescents with autism spectrum disorders in an institution in Bogota, Colombia. Neurología (English Edition), 27(2), 90-96. doi: https://doi.org/10.1016/j.nrl.2011.03.005

Tek, S., \& Landa, R. J. (2012). Differences in autism symptoms between minority and non-minority toddlers. Journal of autism and developmental disorders, 42(9), 1967-1973. doi: https://doi.org/10.1007/s10803-012-1445-8

Villalba, J. A. (2013). Validación del q-chat para detectar el trastorno autista en edades tempranas (Doctoral dissertation, Universidad Nacional de Colombia).

Watson, L. R., Baranek, G. T., Crais, E. R., Reznick, J. S., Dykstra, J., \& Perryman, T. (2007). The first year inventory: retrospective parent responses to a questionnaire designed to identify one-year-olds at risk for autism. Journal of Autism and Developmental Disorders, 37(1), 49-61. doi: https://doi.org/10.1007/ s10803-006-0334-4

Wetherby, A. M., Watt, N., Morgan, L., \& Shumway, S. (2007). Social communication profiles of children with autism spectrum disorders late in the second year of life. Journal of Autism and Developmental Disorders, 37(5), 960-975. doi: https:// doi.org/10.1007/s10803-006-0237-4

Young, R. L., Brewer, N., \& Pattison, C. (2003). Parental identification of early behavioural abnormalities in children with autistic disorder. Autism, 7(2), 125-143. doi: https://doi.org/10.1177/1362361303007002002

Zablotsky, B., Black, L. I., Maenner, M. J., Schieve, L. A., \& Blumberg, S. J. (2015). Estimated prevalence of autism and other developmental disabilities following questionnaire changes in the 2014 National Health Interview Survey. National Health Stat Report, 13(87), 1-20. Disponible en https://www.ncbi.nlm.nih.gov/ pubmed/26632847

Zablotsky, B., Colpe, L. J., Pringle, B. A., Kogan, M. D., Rice, C., \& Blumberg, S. J. (2017). Age of parental concern, diagnosis, and service initiation among children with autism spectrum disorder. American Journal on Intellectual and Developmental Disabilities, 122(1), 49-61. doi: https://doi.org/10.1352/1944-7558-122.1.49

Zwaigenbaum, L., Bryson, S., Rogers, T., Roberts, W., Brian, J., \& Szatmari, P. (2005). Behavioral manifestations of autism in the first year of life. International Journal of Developmental Neuroscience, 23(2), 143-152. doi: https://doi.org/10.1016/j.ijdevneu.2004.05.001 